\title{
Phase field modeling of growth competition of silicon grains
}

\author{
P. Chen, Y.L. Tsai, C.W. Lan* \\ Department of Chemical Engineering, National Taiwan University, No. 1, Sec. 4, Roosevelt Road, Taipei 10617, Taiwan, ROC
}

Received 13 December 2007; received in revised form 18 April 2008; accepted 21 April 2008

Available online 5 June 2008

\begin{abstract}
The growth competition of two grains, with orientations (110) and (100), during directional solidification of silicon is simulated by using a phase field model. The two-dimensional simulations show two distinct competition mechanisms, with either interfacial or kinetic dominance, depending on the undercooling. At low undercooling, the interfacial effect is dominant so that (110) grain grows laterally, expelling the other grain. On the other hand, at high undercooling, the grain competition follows the same pattern at the beginning, but the (100) grain eventually becomes dominant, expanding its domain. In addition, the facet vanishing process and the dihedral angle evolution are discussed. The simulated results and phenomena are consistent with the experimental observations of Fujiwara et al. [Fujiwara K, Obinata Y, Ujihara T, Usami N, Sazaki G, Nakajima K. J Cryst Growth 2004;266:441] and the analytical predictions of Atwater et al. [Atwater HA, Thompson CV, Smith HI. J Mater Res 1988;6:1232].

(C) 2008 Acta Materialia Inc. Published by Elsevier Ltd. All rights reserved.
\end{abstract}

Keywords: Phase field model; Polycrystalline silicon; Solidification; Grain selection

\section{Introduction}

The crystal growth of polycrystalline silicon (poly-Si) from the melt has become an important subject in the photovoltaic industry because of the increasing market share of poly-Si solar cells. Because the grain size and orientation dramatically affect the minority carrier lifetime and thus the conversion efficiency, control of the grain growth behavior, especially grain competition, is critical [1-4]. Fujiwara et al. [1] observed the silicon melt growth starting from a bicrystal seed in (100) and (111) orientations. They found that the cooling rate affected the grain growth behaviors significantly. At low cooling rate $\left(\sim 1 \mathrm{~K} \mathrm{~min}^{-1}\right)$, the planar interface was observed both on (100) and (111) grains. The (111) grain grew laterally to (100) diminishing the (100) grain during the solidification. However, at a much higher cooling rate $\left(\sim 30 \mathrm{~K} \mathrm{~min}^{-1}\right)$, the $(100)$ grain grew faster than the (111) grain and gradually eliminated the (111) grain. Fujiwara et al. [1] proposed that the behavior at the lower cooling case was dominated by the force

\footnotetext{
* Corresponding author. Fax: +8862 23633917.

E-mail address: cwlan@ntu.edu.tw (C.W. Lan).
}

balance at the grain boundary tri-junction; the grain with a lower surface energy will be dragged by the higher one. For the higher cooling case, Atwater et al. [2] proposed the relative growth rates of the grains as the main factor determining growth behavior; the faster grain wins the competition and occupies the space during growth. In addition to the grain competition, in the experiments by Fujiwara et al., macro-steps also appeared from the (100) interface at high cooling rates, while the (111) grain remained planar. These macro-steps on the (100) grain, having various sizes and derivates from the (111) face at unsteady state, grow with time, and only those composed of the (111) face remained at steady state [3]. Simulation of these experiments could be useful, and provide a foundation for the large-scale modeling of poly-Si solidification. However, the simulation of grain growth remains a great challenge in solidification, and no such a simulation showing the key experimental features has been carried out so far.

The phase field model (PFM) has been used extensively to study the microstructural dynamics of solidification (e.g. $[5,6])$, but generally without considering the grain boundary effects. The modeling of polycrystalline material requires the treatment of crystallographic orientations 
and interfacial energies, but very few models have been proposed. Indeed, in polycrystalline phase field modeling, the complicated physical effect of crystalline orientation on solidification and grain interactions needs to be considered. Morrin et al. [7] proposed a free energy density function with $n$ wells for $n$ different orientations. Steinbach et al. [8] developed a multiphase field model and succeeded in modeling the solidification of eutectic and peritectic materials. However, their models were not able to keep the rotation symmetry of the free energy potential in the modeling of grain boundary formation and dynamics. By proposing an orientation field $\theta$, which describes the local grain orientation, Kobayashi et al. $[9,10]$ made a successful attempt, based on a rotation-invariant free energy function, at the phase field modeling of grain boundaries. Warren et al. [11] further extended the model to the solidification of polycrystalline materials. Recently, nucleation phenomena have also been considered by Granasy et al. $[12,13]$. For a material having a highly anisotropic interfacial energy and a tendency to form facets, such as silicon, special attention is needed. By using a regularization method, Eggleston et al. [14] have simulated successfully the crystal shape with corners and edges. Although suitable PFMs have been proposed for the solidification of polycrystalline materials, there are some computational issues. One of the most critical problems in phase field simulation arises from the diffusive interface thickness $\delta$. The choice of $\delta$ needs to be small enough so that the sharp-interface limit can be approached. However, such a value, which is usually of the order of the microscopic capillary length $\mathrm{d}_{0}$, is too small from the simulation point of view. With the use of adaptive meshes [15,16], the computational efficiency can be greatly improved, and this can be particularly useful for polycrystalline solidification having grain boundaries.

In this paper, we present adaptive phase field modeling of poly-Si solidification. During simulation, the mesh is adaptively refined or coarsened with the phase field variable and variable gradients. The directional solidification of silicon from a bicrystal seed is focused, and the effect of undercooling is discussed. The PFM and the adaptive numerical scheme used are described briefly in Section 2 . In Section 3, the validation of the present PFM by an analytical solution is presented through grain boundary groove simulations. Section 4 is devoted to the simulations of the competitive melt growth of silicon, where the analysis and discussions of the growth behaviors are also given. Finally, short conclusions are given in Section 5.

\section{Phase field model}

The present PFM is developed following the work of Warren et al. [11]. The free energy function $F$ in a constant volume $V$ is postulated as

$$
F=\int_{V}\left[f(\phi, T)+\frac{\varepsilon_{\phi}^{2}}{2}|\nabla \phi|^{2}+s \phi^{2}|\nabla \theta|+\frac{\varepsilon_{\theta}^{2}}{2} \phi^{2}|\nabla \theta|^{2}\right] \mathrm{d} V,
$$

where $\phi$ is a phase field variable representing the crystalline state as $\phi=1$ and the melt state as $\phi=0$, and the parameter $\varepsilon_{\phi}$ is used to measure the anisotropic effect. The orientation field variable $\theta$ measures the local orientation of the crystal with respect to the fixed coordinate system, and lies in the domain of $-\pi \leqslant \theta \leqslant \pi$. The Helmholtz free energy density $f(\phi, T)$ takes the form

$f(\phi, T)=p(\phi) \frac{T-T_{m}}{T_{m}}+W \phi^{2}(1-\phi)^{2}$,

where $T$ is the temperature, $T_{\mathrm{m}}$ the melting point, and $W$ is the height of the free energy potential barrier between bulk phases and $p(\phi)=\phi^{3}\left(10-15 \phi+6 \phi^{2}\right)$. The parameter $s$ measures the energy cost due to the misorientation at the grain boundary.

To model the anisotropic crystal shape, the gradient energy coefficient $\varepsilon_{\phi}$ needs to be further modified. As proposed by Kobayashi [17], as a function of the crystalline orientation $\psi$, which is defined as the angle between the interface normal and the reference axis, the coefficient $\varepsilon_{\phi}$ or a crystal with 4-fold symmetry can be written as

$\varepsilon_{\phi}(\psi)=\bar{\varepsilon}_{\phi} \eta(\psi)=\bar{\varepsilon}_{\phi}\left[1+\varepsilon \cos 4\left(\psi-\psi_{0}\right)\right]$.

The crystalline orientation $\psi$ is calculated by $\psi=\tan ^{-1}\left(\phi_{y} / \phi_{x}\right)$, where the subscript in the phase field variable denotes the first derivative with respect to the spatial coordinate. However, when the anisotropic strength $\varepsilon$ is greater than $1 / 15$, missing orientations occur and the crystal shape obtained from the anisotropic function is not realistic where the reciprocal $\gamma$-plot becomes concave. To overcome this, and at the same time to reveal the edges and corners for silicon crystals, we adopt the modified gradient energy coefficient $\varepsilon_{\phi}$ within the missing orientations as proposed by Eggleston et al. [14]:

$\varepsilon_{\phi}=\frac{\varepsilon_{\phi}\left(\psi_{m}\right)}{\cos \left(\psi_{m}\right)} \cos \psi$

where $\psi_{\mathrm{m}}$ is the first missing orientation, and is calculated from the following relationship:

$\eta\left(\psi_{\mathrm{m}}\right) \sin \psi_{\mathrm{m}}+\frac{\partial \eta\left(\psi_{\mathrm{m}}\right)}{\partial \psi} \cos \psi_{\mathrm{m}}=0$.

Finally, through the variation principle that minimizes the total free energy, the evolution of the phase field, orientation field and temperature variables can be derived:

$$
\begin{aligned}
& \frac{\partial \phi}{\partial t}=M_{\phi}\left(\left(\eta \varepsilon_{\phi}\right)^{2} \nabla^{2} \phi+\varepsilon_{\phi}^{2} \frac{\partial}{\partial x}\left(\eta \frac{\partial \eta}{\partial \psi} \frac{\partial \phi}{\partial y}\right)-\varepsilon_{\phi}^{2} \frac{\partial}{\partial y}\left(\eta \frac{\partial \eta}{\partial \psi} \frac{\partial \phi}{\partial x}\right)\right. \\
& -2 W \phi(1-\phi)(1-2 \phi)) \\
& \quad-30 M_{\phi} L \phi^{2}(1-\phi)^{2} \frac{T-T_{\mathrm{m}}}{T_{\mathrm{m}}}-M_{\phi} 2 s \phi|\nabla \theta| \\
& \phi^{2} \frac{\partial \theta}{\partial t}=M_{\theta} \nabla \cdot\left[\phi^{2} \frac{s}{|\nabla \theta|} \nabla \theta\right]-M_{\theta} \varepsilon_{\phi}^{2} \eta \frac{\partial \eta}{\partial \theta}|\tilde{\nabla} \phi|^{2} \\
& \frac{\partial T}{\partial t}=\nabla \cdot(\alpha \nabla T)-30 \phi^{2}(1-\phi)^{2} \frac{L}{C_{\mathrm{p}}} \frac{\partial \phi}{\partial t} .
\end{aligned}
$$


In the above equations, $M_{\phi}$ and $M_{\theta}$ are the mobility of the phase field and the orientation field variables, respectively. $L$ is the latent heat, $C_{\mathrm{p}}$ the heat capacity and $\alpha$ the thermal diffusivity. For simplicity, we also assume equal thermal diffusivity in all phases in the simulation. To link the PFM parameters with the realistic physical properties, the parameters used in the WBM model [5] are taken:

$\varepsilon_{\phi}=\sqrt{6 \sigma \delta}$,

$W=3 \frac{\sigma}{\delta}$,

$M_{\phi}=\frac{\mu_{k} T_{m}}{6 \delta L}$,

where $\sigma$ is the solid-liquid surface energy.

To facilitate the simulation, the governing equations are further represented in dimensionless form, where the length is rescaled by the characteristic length $l_{0}$ and the time $t$ rescaled by $l_{0}^{2} / \alpha$. The dimensionless temperature $u$ is defined by $\left(T-T_{\mathrm{m}}\right) / T_{\mathrm{m}}$ unless otherwise stated. Fig. 1a shows the computational domain of the directional solidification of bicrystalline silicon. A bicrystal seed with different crystalline orientations is placed in front of the undercooled silicon melt. Because the simulation is twodimensional, it is assumed that the (111) and (110) orientations are the same. The symmetry boundary condition is applied to the both sides of the domain, while at the top the temperature is given; the lower thermal boundary is set to be adiabatic. The physical properties of silicon used in the simulation are listed in Table 1. The interface thickness is taken as $4.1 \times 10^{-8} \mathrm{~m}$ in all simulations. According to Wheeler et al. [5], the interface thickness has to be of the a

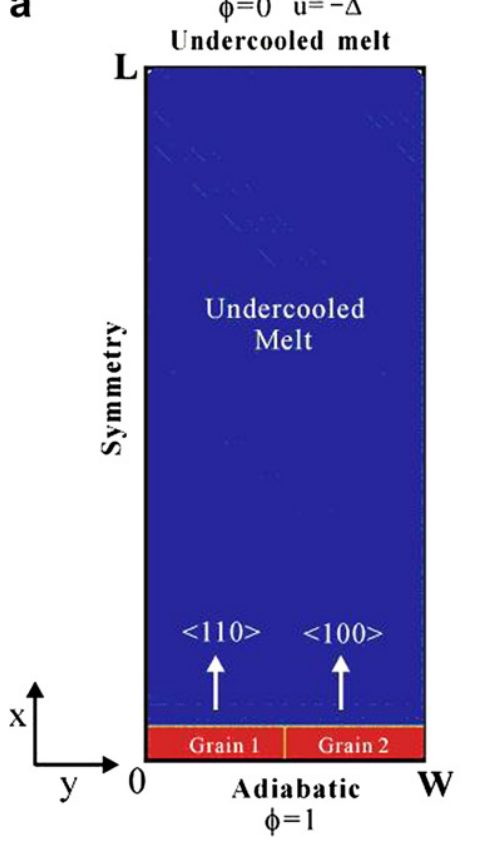

b

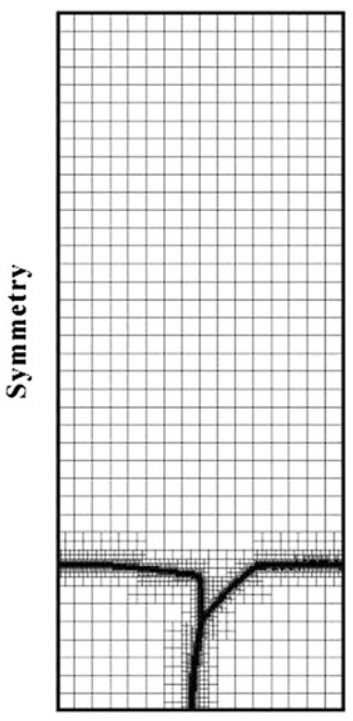

Fig. 1. (a) A schematic directional solidification of silicon bicrystal in undercooled melt. (b) A sample adaptive mesh in silicon growth simulation.
Table 1

Physical properties of silicon

\begin{tabular}{llll}
\hline Properties & Name & Value & References \\
\hline$\alpha$ & Thermal diffusivity $\left(\mathrm{m}^{2} \mathrm{~s}^{-1}\right)$ & $2.8 \times 10^{-5}$ & {$[17]$} \\
$\sigma$ & Surface energy $\left(\mathrm{J} \mathrm{m}^{-2}\right)$ & $0.59^{\mathrm{a}}$ & Estimated \\
$L$ & Latent heat $\left(\mathrm{J} \mathrm{m}^{-3}\right)$ & $4.15 \times 10^{9}$ & {$[17]$} \\
$\mu_{\kappa}$ & Kinetics coefficient $\left(\mathrm{m} \mathrm{K}^{-1} \mathrm{~s}^{-1}\right)$ & $0.02^{\mathrm{b}}$ & Estimated \\
$\varepsilon$ & Anisotropy strength & 0.25 & Estimated \\
\hline
\end{tabular}

a The value of the surface energy is estimated from the equation $\tilde{\gamma}=\sigma\left(V_{\mathrm{m}}^{2}\right)^{1 / 3} / L$ where $\tilde{\gamma}_{\mathrm{Si}}=0.525[15]$.

The kinetic coefficient we used is simply chosen by assumption; however, the order of the value is close to those evaluated from the experiments and the simulations [16-18].

order of capillary length (of the order of $10^{-9} \mathrm{~m}$ ) to approach the sharp-interface solution. However, this will greatly increase the computational cost. Hence, a much thicker interface is adopted, and the comparisons between theory and the simulations thus are merely qualitative. Nevertheless, we anticipate that certain physical features of the experiments could be revealed through the simulation. The governing equations with the boundary conditions are solved by an adaptive finite volume method $[15,16]$. A sample adaptive mesh during simulation is shown in Fig. 1b. As shown, the mesh is refined to give better numerical resolution near the interfaces, while grids are coarser in the area without significant physical features. The criterion for the mesh refinement is based on the phase field variable in the range of $0.05<\phi<0.95$, and the minimum dimensionless mesh size $\Delta x=0.0625$ is used. Before the simulation for silicon melt growth, which will be discussed in Section 4, is presented, we shall first simulate grain boundary grooving problems. Through the comparison with the analytical solutions, the adaptive PFM simulation can be validated.

\section{Grain boundary grooving}

The grain boundary groove is formed during solidification between two adjacent grains. The groove shape at the junction is a force balance of the interfacial tensions. To obtain a static groove shape, a linear temperature profile having a gradient $G$ of $1.25 \times 10^{5} \mathrm{~K} \mathrm{~m}^{-1}$ is given; a domain $16 \mu \mathrm{m} \times 8 \mu \mathrm{m}$ is used. Because once a steady state has been reached, only the static shape is of interest, the release of latent heat does not play a role. The characteristic length $l_{0}$ is $2 \mu \mathrm{m}$ here, which is calculated from $\left(\frac{T_{\mathrm{m} \sigma} \sigma}{G L}\right)^{1 / 2}$ [18]. Two examples, with isotropic and anisotropic interfacial energies, are discussed in the following sections.

\subsection{Dihedral angle}

Again, as just mentioned, the dihedral angle is a result of the force balance at the tri-junction. For simplicity, we have first assumed that the interfacial energy is isotropic. Therefore, the analytical solution of the dihedral angle $\theta_{d}$ could be easily obtained from Young's law as 
$\theta_{\mathrm{d}}=2 \cos ^{-1}\left(\frac{\gamma_{\mathrm{gb}}}{2 \gamma_{\mathrm{sl}}}\right)$

where $\gamma_{\mathrm{gb}}$ is the grain boundary energy and $\gamma_{\mathrm{sl}}$ is the solidliquid surface energy, i.e. $\sigma \cdot \gamma_{\mathrm{gb}}$ can be calculated from the derivation in Ref. [11]:

$\gamma_{\mathrm{gb}}=\frac{\sqrt{W \varepsilon_{\phi}}}{3}\left(1-\phi_{\min }^{3}\right)$,

$\phi_{\min }=\frac{1}{\left(1+\frac{s}{\varepsilon_{\phi}} \Delta \theta\right)}$.

Three cases with different grain boundary energies are simulated: (a) $\gamma_{\mathrm{gb}}=\gamma_{\mathrm{sl}}$, (b) $\gamma_{\mathrm{gb}}=1.5 \gamma_{\mathrm{sl}}$ and (c) $\gamma_{\mathrm{gb}}=1.95 \gamma_{\mathrm{sl}}$. In addition, to determine the dihedral angle from simulated results, the position of $\phi=0.5$ is taken for measuring the angle; the dihedral angle is obtained from the intersection of the grains. Fig. 2 shows the simulation results, and the
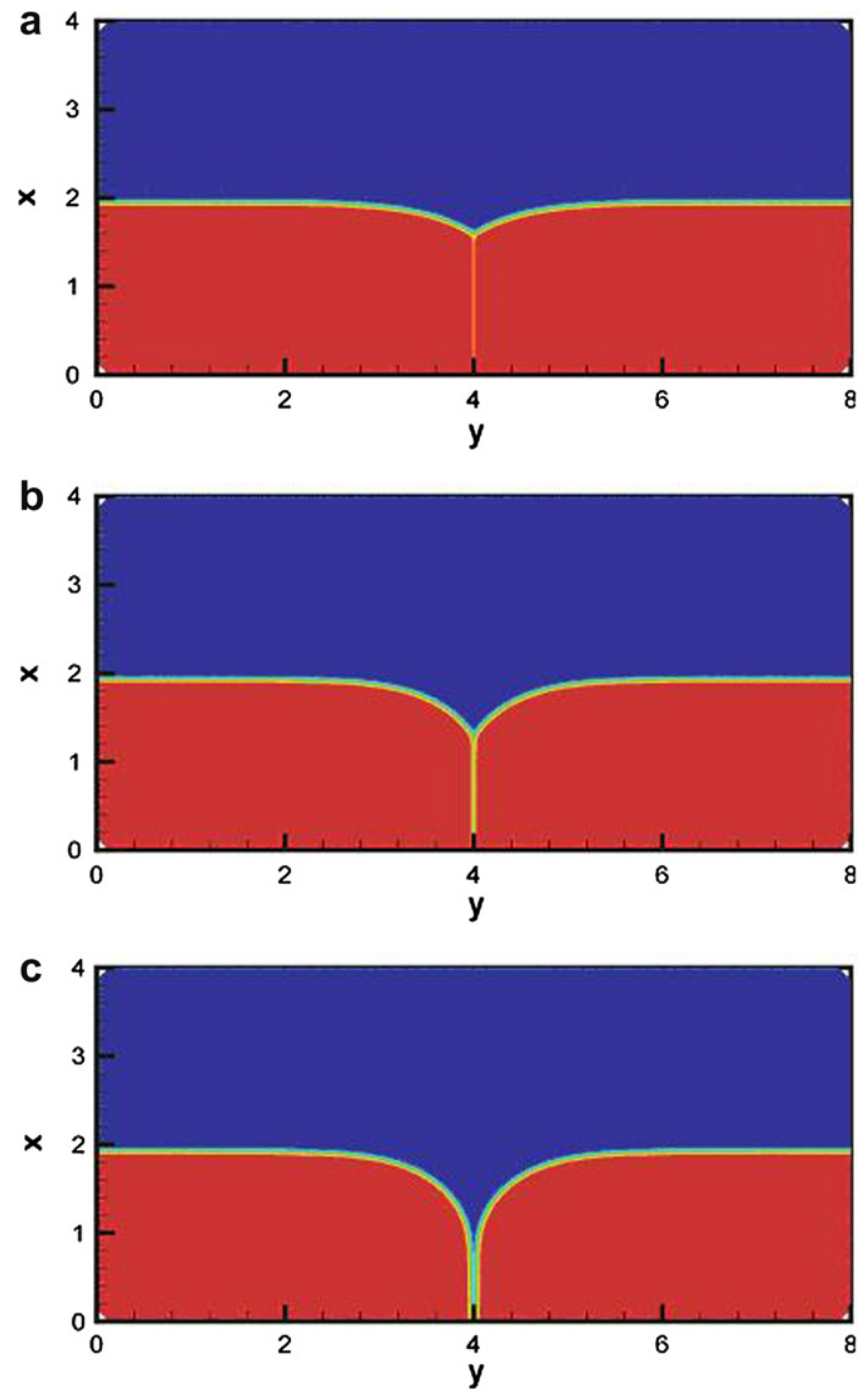

Fig. 2. Simulations of isotropic grain boundary groove: (a) $\gamma_{\mathrm{gb}}=\gamma_{\mathrm{sl}}$, (b) $\gamma_{\mathrm{gb}}=1.5 \gamma_{\mathrm{sl}}$, (c) $\gamma_{\mathrm{gb}}=1.95 \gamma_{\mathrm{sl}}$. In each figure, melt is at the upper half and the crystals are at the lower half. As predicted by Young's law, the dihedral angle will decrease as the grain boundary energy increases.
Table 2

Comparison of calculated dihedral angles with analytical solutions

Dihedral angle comparison

\begin{tabular}{llll}
\hline$\gamma_{\mathrm{gb}} / \gamma_{\mathrm{sl}}$ & 1 & 1.5 & 1.95 \\
Analytical & $118^{\circ}$ & $78^{\circ}$ & $25^{\circ}$ \\
Simulation & $119^{\circ}$ & $76^{\circ}$ & $24^{\circ}$ \\
\hline
\end{tabular}

comparison of the calculated dihedral angles with the analytical solutions is shown in Table 2. As shown, they are in good agreement; the trivial difference is due to the diffusive interface assumed in the PFM.

\subsection{Anisotropic grain boundary groove}

We have obtained a good agreement between the simulated results and the analytical ones for the isotropic grain boundary energy. We now need to further check the agreement for the case with highly anisotropic surface energy. The analytical solution for the grain boundary groove with highly anisotropic surface energy is also available [18] as the following:

$$
\begin{aligned}
& x=\frac{T_{\mathrm{m}}}{G L} \int_{\theta_{\mathrm{d}}}^{\psi}\left(\gamma_{\mathrm{sl}}+\frac{\partial^{2} \gamma_{\mathrm{sl}}}{\partial \psi^{2}}\right) \frac{\sin \psi}{y} \mathrm{~d} \psi, \\
& y=-\frac{2 T_{\mathrm{m}}}{G L}\left(\gamma_{\mathrm{sl}} \sin \psi+\frac{\partial \gamma_{\mathrm{sl}}}{\partial \psi} \cos \psi-\left.\gamma_{\mathrm{sl}}\right|_{\psi=\frac{\pi}{2}}\right)^{1 / 2} .
\end{aligned}
$$

The above equations are solved in two intervals, i.e. $\theta_{\mathrm{d}} \leqslant \psi \leqslant \theta_{1}$ and $\theta_{2} \leqslant \psi \leqslant \frac{\pi}{2}$, where $\theta_{1}$ and $\theta_{2}$ are orientations very close to the kink orientation. In Eq. (15), the dihedral angle $\theta_{\mathrm{d}}$ can be obtained from Herring's relation [19]:

$\gamma_{\mathrm{gb}}-2 \frac{\partial \gamma_{\mathrm{sl}}}{\partial \psi} \sin \psi-2 \gamma_{\mathrm{sl}} \cos \psi=0$

In order to prevent the tilting of the grain boundary due to unequal horizontal forces at the junction, the crystal orientations of the grains are chosen to have the same value but with an opposite sign. Three cases with different crystal orientation $\psi_{0}$ are simulated: $\pi / 6, \pi / 4$ and $\pi / 3$. The grain boundary energy is fixed at $1.99 \gamma_{\mathrm{sl}}$, so that only the surface energy is varied for all three cases. The anisotropic strength $\varepsilon=0.08$ is adopted. The reason for not using a higher $\varepsilon$ as listed in Table 1 is to avoid the oscillating wave, so that a static shape can be obtained by simulation for comparison. Fig. 3 shows a comparison of the simulated results with the analytical solutions. Again, the calculated groove shapes are in good agreement with the analytical ones. This good agreement reinforces our confidence in the simulation of bicrystal problems.

\section{The competitive growth of silicon grains}

The simulation of the competitive growth of silicon grains, as illustrated in Fig. 1a, is presented in this section. The following values are chosen: coupling constant 


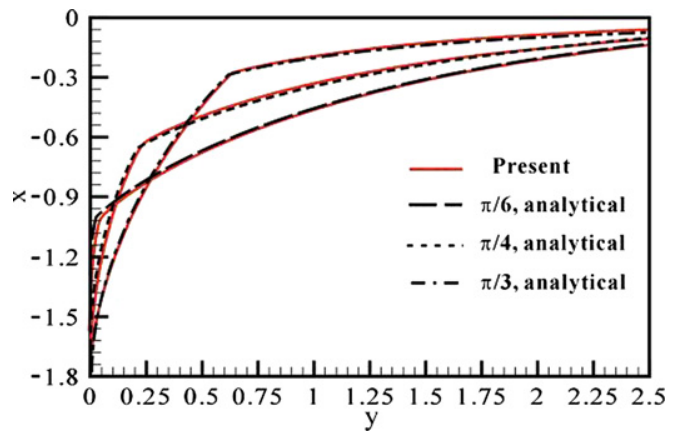

Fig. 3. Comparison of calculated anisotropic grain boundary groove shape with analytical solution at $\varepsilon=0.08$.

$s=2 \mathrm{~J} \mathrm{~m}^{-2}$ and characteristic length $l_{0}=2.1 \times 10^{-6} \mathrm{~m}$; the domain size is taken as $42 \mu \mathrm{m} \times 16.8 \mu \mathrm{m}$. In addition, the orientation field mobility $M_{\theta}$ is set to $0.05 M_{\phi}$, because the time scale of solidification is much smaller than the grain boundary dynamics. In other words, evolution of the crystal orientation is much slower than solidification. It should be pointed out that the domain size and the simulation time are three orders of magnitude smaller than those in the real experiments [1]. In view of the diffusive interface kinetics, the characteristic time for silicon growth is about $10^{-7} \mathrm{~s}$; therefore it is too costly to simulate the real experiments, which lasted a few minutes. In fact, since the physical features are the focus of this study, simulation using smaller length and time scales should reveal the same physical phenomena. Two undercoolings, $\Delta=1.685 \mathrm{~K} / \mathrm{T}_{\mathrm{m}}$ and $\Delta=6.74 \mathrm{~K} / \mathrm{T}_{\mathrm{m}}$, are simulated, and the simulated results for the evolution of grain growth are shown in Figs. 4 and 5, respectively. As shown, the interface morphology and the grain competition are consistent with the observed ones [1]; the (110) grain prevails at low undercooling, while the $(100)$ grain becomes dominant at high undercooling. Further discussion of the morphology and grain competition is given in the following sections.

\subsection{Morphology}

The evolution of morphology for $\Delta=1.685 \mathrm{~K} / \mathrm{T}_{\mathrm{m}}$ and $\Delta=6.74 \mathrm{~K} / \mathrm{T}_{\mathrm{m}}$ extracted from Figs. 4 and 5 are put together for comparison in Fig. 6a and b, respectively. As shown, with a smaller undercooling at $\Delta=1.685 \mathrm{~K} / \mathrm{T}_{\mathrm{m}}$, the interface of the (110) grain remains planar, but small edges emerge from the $(100)$ interface. The difference in

\section{Undercooling $=1.685 \mathrm{~K}$}
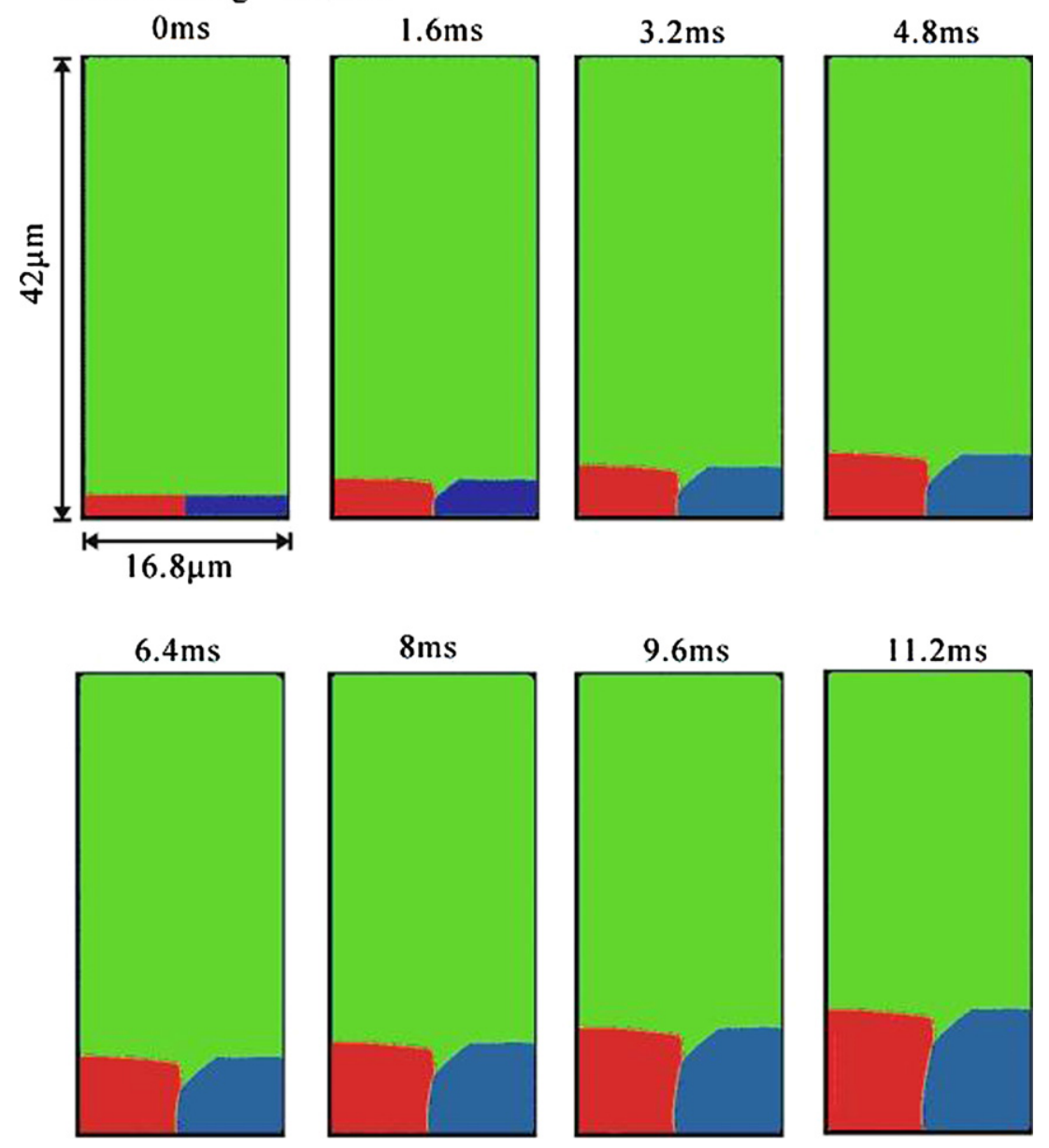

Fig. 4. Simulation of Si melt growth at $\Delta=1.685 \mathrm{~K} / \mathrm{T}_{\mathrm{m}}$. 


\section{Undercooling $=6.74 \mathrm{~K}$}
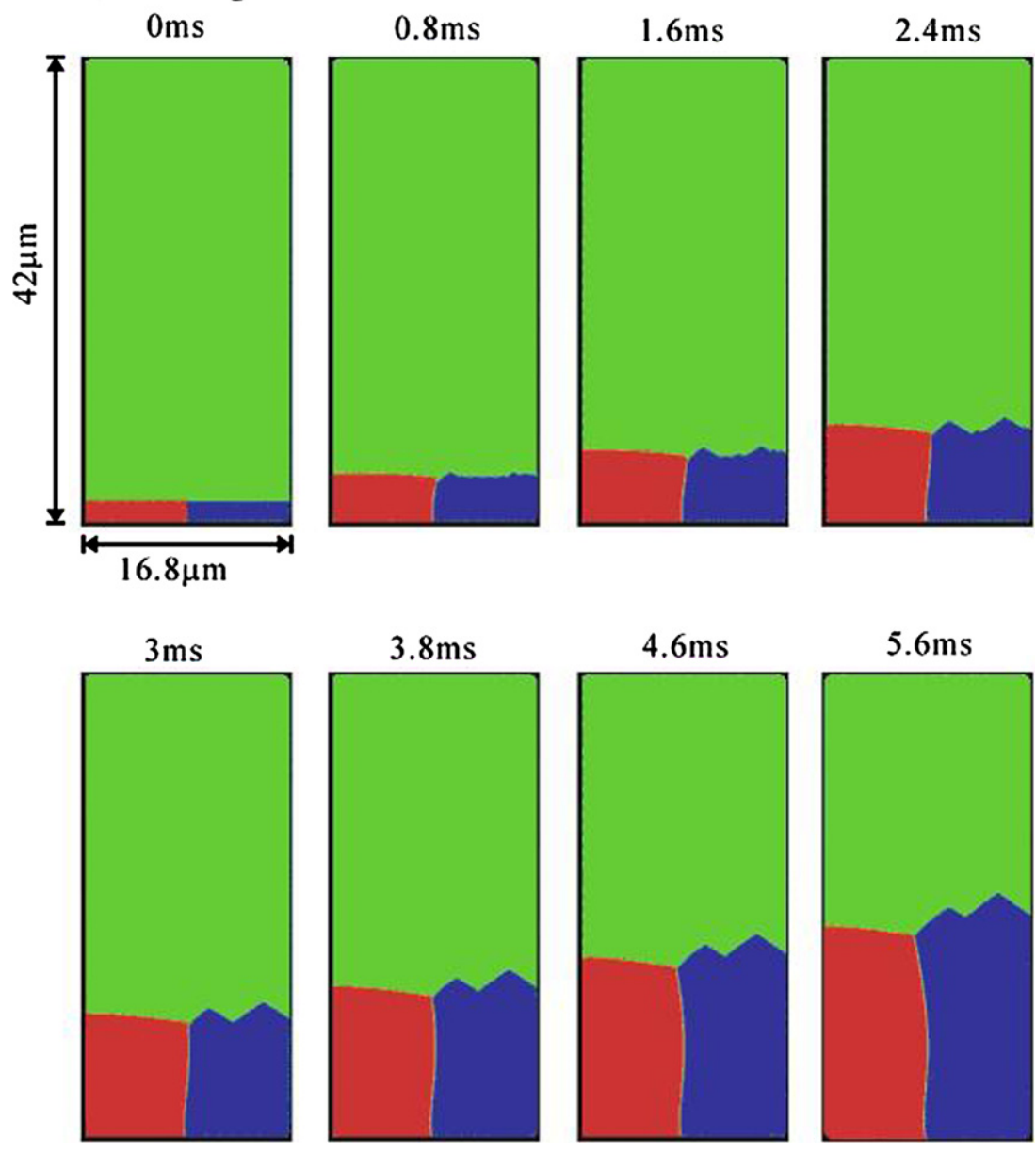

Fig. 5. Simulation of Si melt growth at $\Delta=6.74 \mathrm{~K} / \mathrm{T}_{\mathrm{m}}$.
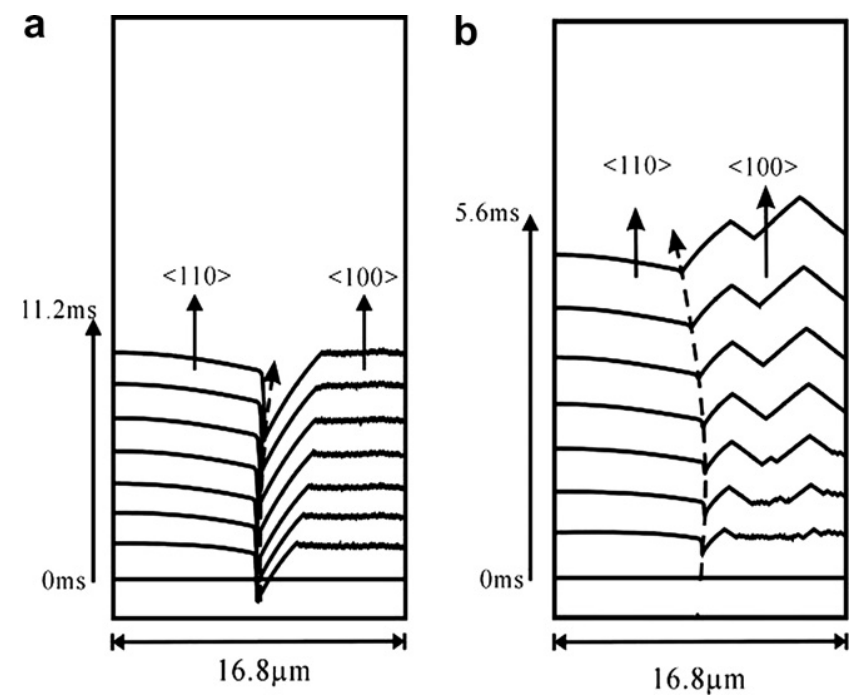

Fig. 6. Evolution of the morphology of competitive growth at different undercoolings: (a) $1.685 \mathrm{~K} / \mathrm{T}_{\mathrm{m}}$ and (b) $6.74 \mathrm{~K} / \mathrm{T}_{\mathrm{m}}$.

the morphology can be explained by the grain surface energy, which stabilizes the solid-liquid interface. Apparently, the (110) surface energy plays a stronger role in stabilizing the interface. These edges appearing in the (100) surface are smaller than $1 \mu \mathrm{m}$, and the sizes remain about the same during solidification at $\Delta=1.685 \mathrm{~K} / \mathrm{T}_{\mathrm{m}}$. However, the situation is slightly different for $\Delta=6.74 \mathrm{~K} / \mathrm{T}_{\mathrm{m}}$ in that these edges coarsen into larger facets. Furthermore, the intersection angle of these edges is also changing during solidification. As the edges become larger, only those with an angle close to $70^{\circ}$ remain, as shown in Fig. 7a. This could be explained by the minimization of the surface energy. When the missing orientations disappear, the equilibrium edge angle is obtained. This can be further explained by the equilibrium crystal shape grown from a seed in an undercooled melt; the anisotropic strength $\varepsilon$ is greater than $1 / 15$, so that the edge and angle appear in the range of the missing orientations [14]. Fig. 7b shows a sample simulation of the equilibrium crystal shape. As shown, its equilibrium edge angle is about $70^{\circ}$.

Interestingly, the vanishing of the facet, leading to big (110) facets, or the coarsening process, is suppressed by the surface energy at low undercooling, but becomes significant at high undercooling. This is consistent with the experimental observations. Nevertheless, it should be pointed out that the crystal shape with edges obtained 
a
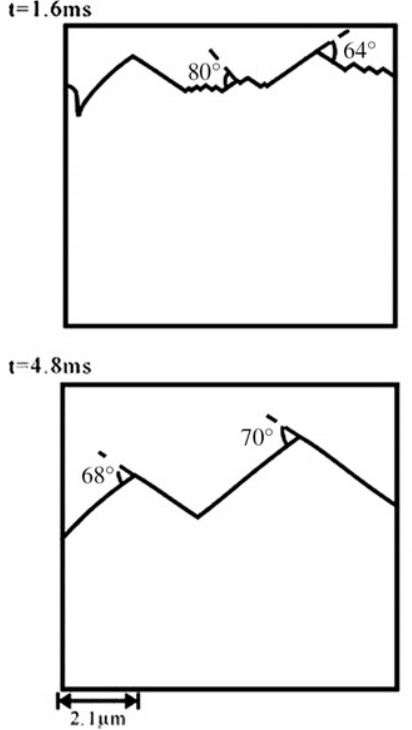

b

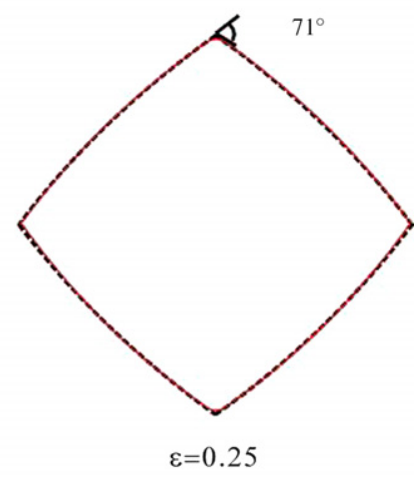

Fig. 7. (a) Coarsening of edges at $\Delta=6.74 \mathrm{~K} / \mathrm{T}_{\mathrm{m}}$. (b) Equilibrium shape of the crystal at $\varepsilon=0.25$.

using the present anisotropic function, though similar to the experimental results, is not the case for the faceted morphology; they are not totally flat surfaces. According to Wulff's theorem, the faceted portion of the crystal corresponds to the cusps in the polar plot of the surface energy, but the function we use here is a smooth function. In other words, the simulation based on the smooth function leads to curved edges instead of facets. However, the curved surface at high anisotropic strength looks quite similar to a facet.

\subsection{Force balancing at the groove root}

According to Fujiwara et al. [1], growth behavior at low undercooling is dominated by the force balance at the grain boundary root. In other word, the grains with lower surface energies remain. To check this, we apply Herring's relation [19] to analyze the force balance in the groove during growth at $\Delta=1.685 \mathrm{~K} / \mathrm{T}_{\mathrm{m}}$. Herring's relation describes the interfacial tension balance at the junction for anisotropic interfacial energies:

$\gamma_{\mathrm{gb}} \cos \beta=\sum_{i=1}^{2}\left(\gamma_{i} \sin \psi_{i}-\frac{\partial \gamma_{i}}{\partial \psi_{i}} \cos \psi_{i}\right)$,

$\gamma_{\mathrm{gb}} \sin \beta=\sum_{i=1}^{2}\left(\gamma_{i} \cos \psi_{i}+\frac{\partial \gamma_{i}}{\partial \psi_{i}} \sin \psi_{i}\right)$.

In the above equations, the grain boundary energy is assumed to be isotropic, where the angle $\beta$ is defined as the tilt angle of the grain boundary with the $x$-axis. It should be pointed out that by definition the interfacial tension is equivalent to the surface energy. For the horizontal part of the force, we have taken the force as positive when it is pointing to the $y$-axis, which here is the direction of the (100) interfacial tension. For the vertical part, the force is positive when it is towards the positive $x$-axis. The overall interfacial tension is defined by $|\Sigma F|=\sqrt{F_{\mathrm{h}}^{2}+F_{\mathrm{v}}^{2}}$, where $F_{\mathrm{h}}$ and $F_{\mathrm{v}}$ are the overall horizontal and vertical interfacial tensions, respectively. The decrease in the overall interfacial tension, in other words, corresponds to the minimization of the surface energy.

Fig. 8 shows the overall horizontal and vertical interfacial tension evolutions at $\Delta=1.685 \mathrm{~K} / \mathrm{T}_{\mathrm{m}}$; the total interfacial tension is also included. As shown, the overall horizontal tension is much greater than zero, indicating that the interfacial tension on the (100) face is significantly larger than that on the (111) face. The value is decreasing with time as force relaxes, consistent with the experimental observation [1]. For the overall vertical interfacial tension, the value reduced to about $-0.6 \mathrm{~J} \mathrm{~m}^{-2}$ (in the negative $x$ direction). This can be easily understood in terms of the reluctance of the grains to grow, so that the interfacial tension is a suppressing force. However, because the undercooling is sufficiently large, solidification still proceeds [14]. Nevertheless, from the plot of the overall interfacial tension shown in Fig. 8, the value is decreasing with time. Since the thermal driving force remains approximately the same in the simulation, the horizontal interfacial is indeed the key factor in determining the trend of the tension evolution. In other word, the decrease in the interfacial tension indicates the minimization of the surface energy. Eventually, the gain having a lower surface energy becomes dominant in the growth, while the groove shape reaches an equilibrium.

A similar interfacial force balance is also carried for high undercooling at $\Delta=6.74 \mathrm{~K} / \mathrm{T}_{\mathrm{m}}$, and the interfacial tension balance results are given in Fig. 9, again for the component and overall interfacial tensions. As shown in Fig. 9, the evolution of the interfacial force balances is quite different from the previous one. In Fig. 9, the horizontal and overall interfacial tensions increase at the beginning, and then gradually decrease. According to the discussion in Ref. [2], instead of the interfacial tension balance at the groove, the growth at high undercooling is dominated by the relative growth rates among the grains. Hence, we should first

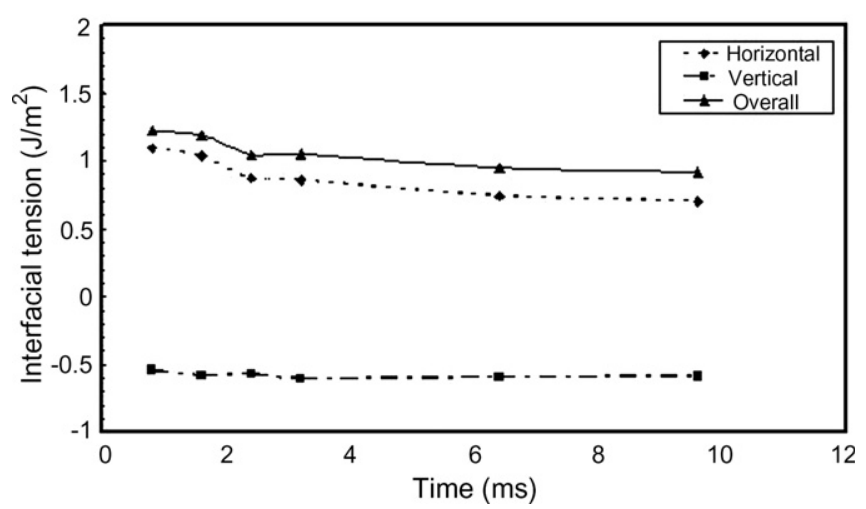

Fig. 8. Plot of the overall force component vs. time at $\Delta=1.685 \mathrm{~K} / \mathrm{T}_{\mathrm{m}}$ 


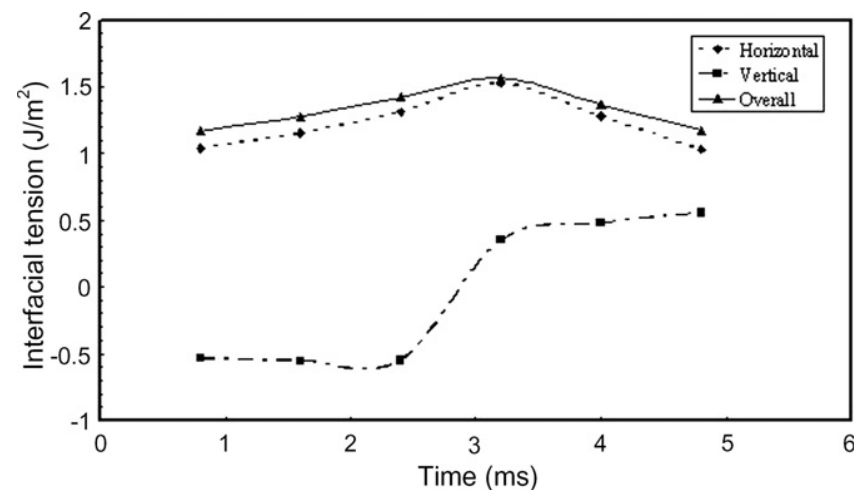

Fig. 9. Plot of the overall force component vs. time at $\Delta=6.74 \mathrm{~K} / \mathrm{T}_{\mathrm{m}}$.

address the growth rate anisotropy in the present PFM. According to the work by Kobayashi [17], modifying the gradient energy coefficient as an orientation function in the PFM also leads to an anisotropic kinetic coefficient, i.e.

$\mu_{k}=\frac{6 M_{\phi} \varepsilon_{\phi} L}{T_{\mathrm{m}} \sqrt{2 W}} \eta(\psi)$.

For a crystal with 4-fold symmetry in two-dimensions, the anisotropic kinetic coefficient is maximum in the (100) direction $\left(\theta=0^{\circ}\right)$ and minimum in the (110) direction $\left(\theta=45^{\circ}\right)$. Atwater et al. [2] further explained that the growth direction of the grain boundary could be found from the anisotropic growth rates:

$\sin \alpha=\frac{v_{\text {slower grain }}}{v_{\text {faster grain }}}$,

where $\alpha$ is the angle between the grain boundary and the slower growing grain surface. For our case here, initially the growth is affected by the interfacial tension balance so that the grain boundary is pulled toward the right as in the case of low undercooling. However, since the effect of kinetics becomes significant at high undercooling, the faster growing grain $(\langle 100\rangle)$ forces the dihedral angle to decrease. As a result, the resultant interfacial tension increases, instead of relaxing, until the grain boundary is tilted toward the (110) grain. The tilting to the left approximately follows the relationship in Eq. (21). Once the grain boundary tilting angle $\alpha$ is established, the interfacial tension start to relax and eventually an equilibrium groove will form.

We also confirm this via force balance analysis of the experimental results in Ref. [1]. For the growth experiment at a cooling rate of $30 \mathrm{~K} \mathrm{~min}^{-1}$, the growth rate of each grain was approximately constant, so that the growth direction of the grain boundary remained about the same. By applying Herring's relation to the experimental results, we find that the overall interfacial tension decreases from about 0.7 to $0.15 \mathrm{~J} \mathrm{~m}^{-2}$ within the growth period. Therefore, we can conclude that the interfacial tension balance at $\Delta=6.74 \mathrm{~K} / \mathrm{T}_{\mathrm{m}}$, initially is destroyed by the kinetic effect and the interfacial tension balancing restarts when the kinetic effect no longer dominates the growth behavior.
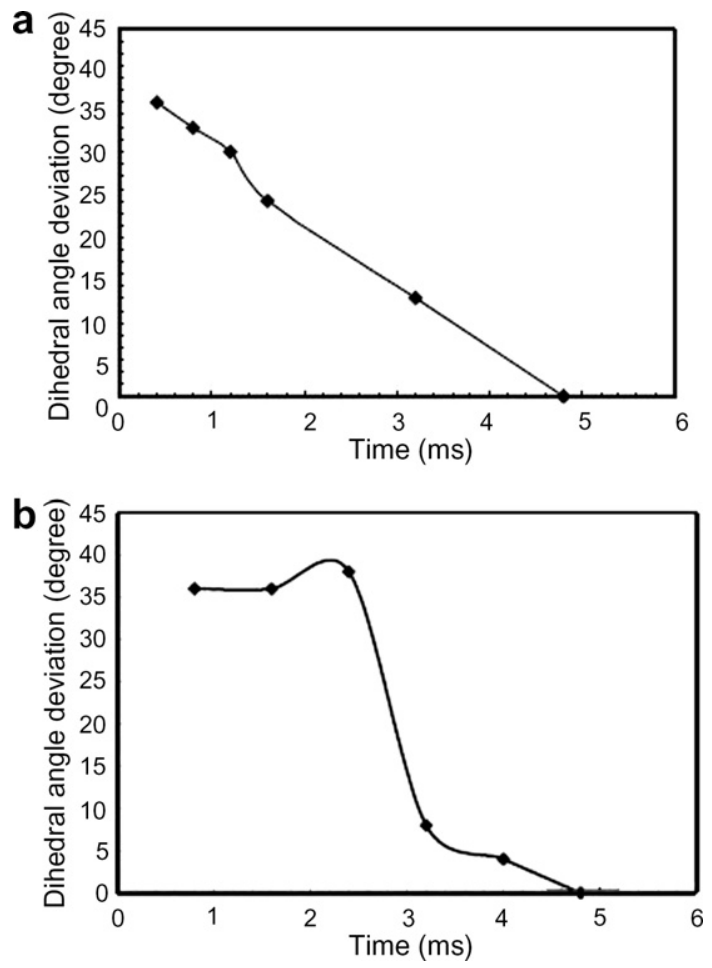

Fig. 10. Evolutions of the dihedral angle deviation from the final state: (a) low undercooling at $1.685 \mathrm{~K} / \mathrm{T}_{\mathrm{m}}$ and (b) high undercooling at $6.74 \mathrm{~K} / \mathrm{T}_{\mathrm{m}}$.

Furthermore, the evolution of the dihedral angle can also be examined. As we just illustrated, the interface energy eventually decreases with time in both cases. In other words, the dihedral angle between two grains should also reach an equilibrium. Since, we have no actual balance angle, we take the final one at the end of simulation as a reference. Then, the evolutions of the angle deviating from the final one can be examined as shown in Fig. 10a and b for low and high undercoolings respectively. For low undercooling $\left(\Delta=1.685 \mathrm{~K} / \mathrm{T}_{\mathrm{m}}\right)$, since the total process is dominated by the interfacial energy, the same trend is found for the angle deviating from the final one as shown in Fig. 10a. This is also consistent with the experimental observation in Ref. [1]. On the other hand, for the high undercooling case $\left(\Delta=6.74 \mathrm{~K} / \mathrm{T}_{\mathrm{m}}\right)$ as shown in Fig. 10b, at the beginning, the balance is destroyed by the kinetic effect. However, once the grain competition has reached to a certain extent, the angular deviation from the final state starts to decrease, showing the same trend as the total interfacial energy after the peak.

\section{Conclusions}

In this study, phase field simulation is carried out for the competitive growth of silicon grains. The orientation field variable is used to treat polycrystalline solidification, and a high anisotropic function is adopted to simulate the edges. The present model is first validated through grain boundary groove simulations. The force balance test and the equilibrium groove shape from the simulation are in good agree- 
ment with the analytical results. Furthermore, competitive growth of silicon grains is simulated, and the competition between the interfacial and kinetic effects is illustrated. The undercooling is found to be the most important factor for the competition and the simulation results, which are consistent with the experimental observation and model predictions. Although the present phase field simulation is primitive and qualitative, it indeed provides deeper physical insights into the polycrystalline crystal growth and grain control.

\section{References}

[1] Fujiwara K, Obinata Y, Ujihara T, Usami N, Sazaki G, Nakajima K. J Cryst Growth 2004;266:441.

[2] Atwater HA, Thompson CV, Smith HI. J Mater Res 1988;6:1232.

[3] Fujiwara K, Nakajima K, Ujihara T, Usami N, Sazaki G, Hasegawa H, et al. J Cryst Growth 2002;243:275.

[4] Aoyama T, Kuribayashi K. Acta Mater 2000;48:3739.
[5] Wheeler AA, Boettinger WJ, MacFadden GB. Phys Rev A 1992;45:7424.

[6] Loginova I, Amberg G, Argen J. Acta Mater 2001;49:573.

[7] Morin B, Elder KR, Sutton M, Grant M. Phys Rev Lett 1995;75:2156.

[8] Steinbach L, Pezzola F, Nestler B, Sesselberg M, Prieler R, Schmitz GJ, et al. Physica D 1996;94:135.

[9] Kobayashi R, Warren JA, Carter WC. Physica D 1998;119:415.

[10] Kobayashi R, Warren JA, Carter WC. Physica D 2000;140:141.

[11] Warren JA, Kobayashi R, Lobkovsky AE, Carter WC. Acta Mater 2003;51:6035.

[12] Granasy L, Borzsonyi T, Pusztai T. Phys Rev Lett 2002;88:206105.

[13] Granasy L, Borzsonyi T, Pusztai T. Phys Rev Lett 2007;98:035703.

[14] Eggleston JJ, McFadden GB, Voorhees PW. Physica D 2001;150:91.

[15] Lan CW, Liu CC, Hsu CM. J Comp Phys 2002;178:464.

[16] Lan CW, Hsu CM, Chang YC. Phys Rev E 2002;65:61601.

[17] Kobayashi R. Physica D 1993;63:410.

[18] Voorhees PW, Coriell SR, McFadden GB. J Cryst Growth 1984;67:425.

[19] Herring C. The physics of powder metallurgy. New York: McGrawHill; 1951. p. 143. 